\title{
Hypothalamic Noradrenergic Hyperactivity and Detrimental Bone Status in an Animal Model of Nutritional Growth Retardation
}

\author{
María I. Olivera ${ }^{1}$, Gabriela E. Compagnucci ${ }^{1}$, Cecilia V. Compagnucci ${ }^{1}$, Christian E. Lezón ${ }^{1}$, \\ Patricia Mandalunis ${ }^{2}$, Sandra I. Hope ${ }^{3}$, Liliana G. Bianciotti ${ }^{4}$, Juan C. Elverdin ${ }^{1}$, Rosa M. Alippi ${ }^{1}$, \\ Marcelo S. Vatta ${ }^{3}$ and Patricia M. Boyer*,1
}

\author{
${ }^{I}$ Department of Physiology, School of Dentistry, University of Buenos Aires, Argentina \\ ${ }^{2}$ Department of Histology and Embriology, School of Dentistry, University of Buenos Aires, Argentina \\ ${ }^{3}$ Department of Physiology (IQUIMEFA-CONICET), School of Pharmacy and Biochemistry, University of Buenos \\ Aires, Argentina \\ ${ }^{4}$ Department of Pathophysiology, School of Pharmacy and Biochemistry, University of Buenos Aires, Argentina
}

\begin{abstract}
We have studied hypothalamic noradrenergic activity in relation with bone status in a nutritional growth retardation model (ND). Control rats (C) were fed ad libitum. ND received $80 \%$ of the diet consumed by $\mathrm{C}$ for 4 weeks and later refed ad libitum for 8 weeks. Food restriction induced detrimental effects on body and femur weight and length $(P<0.05)$ and bone biomechanical properties $(P<0.001)$. Thickness of proliferative and hypertrophic zone $(\mu \mathrm{m})$ of growth plate cartilage and bone volume $(\%$, mean \pm SE) were $225.96 \pm 5.70$ v. $280.70 \pm 12.52,95.16 \pm 5.81$ v. $134.60 \pm 9.30$, $17.64 \pm 3.23$ v. $26.80 \pm 2.03$, respectively $(P<0.05)$; anterior and posterior hypothalamus norepinephrine uptake and release and tyrosine hydroxylase activity $(\%$ of control) were $79.05 \pm 3.56,67.00 \pm 10.00,164.26 \pm 16.58$ and $80.65 \pm 5.92$, $147.00 \pm 1.00,152.42 \pm 9.30$, respectively $(P<0.05)$. Thus, impaired biomechanical bone performance in ND could be due, in part, to the increased hypothalamic noradrenergic activity in response to restriction. Normalization of parameters with refeeding suggests no long-term side-effects in undernourished rats.
\end{abstract}

Keywords: Nutritional growth retardation, bone status, hypothalamic noradrenergic activity.

\section{INTRODUCTION}

Suboptimal and chronic energy intake results in physiological, metabolic, cellular and behavioral responses considered to be adaptive mechanisms to caloric restriction [1]. In humans, certain parental misconceptions, health practices and beliefs concerning what constitutes a healthy diet for infants can restrict their nutrition to the point of inducing a nutritional disease known as nutritional dwarfing (ND) [2]. Although poverty and food shortage in developing countries remain the most common cause of $\mathrm{ND}$, inappropriate eating habits can also lead to children's failure to thrive among the middle-to-upper socio-economic class $[3,4]$. ND refers to a pattern of growth defined by the anthropometric indices of the Wellcome classification system [5], characterized by subnormal body and length growth where weight-for-height deficit and alterations in the biochemical markers of malnutrition are not evident $[6,7]$.

In our laboratory we have developed a nutritional stress model in weanling male rats placed on a $20 \%$ restricted balanced diet for a long time (ND rats), that closely resembles the sub-optimal nutrition observed in children who consume inappropriate diets with insufficient total energy to sustain normal growth and weight gain [8]. The nutritional status model is based on clinical paediatric findings related to

*Address correspondence to this author at the Department of Physiology, School of Dentistry, University of Buenos Aires, Argentina;

E-mail: pboyer@fisio.odon.uba.ar infant feeding practices such as hypocaloric foods that are given to infants postlactation $[9,10]$.

In a previous study performed in our laboratory, [11] we observed inhibition of somatotrophic and reproductive axes in this nutritional stress model in post-weaning male rats that could be due, at least in part, to a decrease in serum leptin levels, an adipocyte-derived hormone linked to somatic growth, metabolism and reproduction [12-14]. These physiological adaptations could be considered as survival advantages during periods of sub-optimal energy intake, ensuring substrate fluxes to issues that require energy constantly (i.e. brain). In fact, when energy intake is limited, serum leptin levels fall signals the brain to initiate responses including reduction in body growth, delay in puberty onset and fertility, suppression of metabolic pathways and activation of the hypothalamic-pituitary-adrenal axis (HPA), all survival responses that reduce the risk of morbidity and death [15]. However, this adaptive responsed to caloric restriction would be predicted to be deleterious to bone status. In fact, previous studies performed in our laboratory showed impaired mechanical femoral competence in ND rats that may result from altered bone mass and architectural distribution rather than its intrinsic quality, a suggestion supported by the high correlation found between bone mineral content and bone mineral density $v$. body weight, and ultimate load and yielding load v. moment of inertia $[16,17]$.

During adaptive behavioral and physical changes in order to face stressors, activation of the stress system occurs. The hypothalamus, in particular the noradrenergic system, is an 
important mediator in adaptive response during stress. Locus ceruleus-norepinephrine/autonomic sympathetic neurons of the hypothalamus and brain stem in association with corticotropin-releasing hormone/arginine-vasopressin respectively regulate the peripheral activities of HPA axis and the systemic/adreno-medullary sympathetic nervous systems, in order to maintain homeostasis [18].

As ND animal is a nutritional stress model and hypothalamus is an integrative centre involved in the control neuroendocrine function as well as energetic metabolism [1922], where somatic growth and bone status are sensed [2224], the aim of the present work was to study the hypothalamic noradrenergic activity profile, neuronal norepinephrine (NE) uptake and release and tyrosine hydroxylase (TH) activity, in relation with bone status in a nutritional growth retardation model induced by $20 \%$ reduction of food intake, started immediately after the weaning period. Parameters mentioned above were also studied during refeeding.

\section{MATERIALS AND METHODOLOGY}

\section{Animals}

Weanling male Wistar rats (21-23 days old; mean initial body weight $47.20 \pm 2.48 \mathrm{~g}$ ) were provided by the Animal Resources of the Department of Biochemistry, School of Dentistry, University of Buenos Aires, Argentina. Animals were housed and kept under $12 \mathrm{~h}$ light-12 $\mathrm{h}$ dark cycles. Room temperature was maintained at $21 \pm 1{ }^{\circ} \mathrm{C}$ with $50-60 \%$ humidity. The experiment was conducted in accordance with the principles and procedures outlined in the National Institutes of Health Guide for the Care and Use of Laboratory Animals [25] and approved by the University of Buenos Aires Ethic Committee.

\section{Diet}

Animals were fed with a standard diet (Purina chow) of the following composition $(\mathrm{g} / 100 \mathrm{~g})$ : protein, 22.7; lipids, 7.09; fibre, 6.0; Ca, 1.3; P, 0.8; ashes, 5.01; water, 7.60; dextrin, balance.

\section{Experimental Design}

Sixty rats were randomly assigned to two groups of 30 animals each one: control (C) and nutritional dwarfing (ND). $\mathrm{C}$ rats were fed freely with the standard diet. ND rats received, for 4 weeks, $80 \%$ of the amount of food consumed by $\mathrm{C}$ the previous day, corrected by body weight (food intake in $\mathrm{g} / 100 \mathrm{~g}$ body weight per day). After 4 weeks of food restriction, the ND group was fed ad libitum for 8 weeks with the same standard diet given to the $\mathrm{C}$ group. All rats had free access to water. Body weight was recorded every day in the morning before food distribution, and length, every $4 \mathrm{~d}$. Growth data were compared over time. Dietary intake was registered daily. A group of animals was sacrificed under anaesthesia after 4 weeks of food restriction (w4) and after 8 weeks of refeeding, when ND rats reached controls anthropometric parameters (w12). After removal of the brain, hypothalamus was dissected in anterior $(\mathrm{AH})$, medium $(\mathrm{MH})$ and posterior $(\mathrm{PH})$ sections to determine noradrenergic activity. Immediately, both femurs and tibiae from each animal were dissected avoiding periostial lesion and weighed, and their length was determined with a digital calliper. Femurs were used for mechanical studies whereas tibiae for histomorphometric studies. Additionally, ten rats were sacrificed for initial measurements on the day the experiment began (day 0 ).

\section{Nutritional Status}

\section{Anthropometry}

Body weight and length were recorded serially during the experimental period (daily and $4 \mathrm{~d}$, respectively), after a 2-4 $\mathrm{h}$ fasting period. A Mettler PC 4000 scale (Zurich, Switzerland) was used to measure body weight with an accuracy of \pm 1 g. For length measurements, animals were anaesthetized slightly with diethyl ether in an anaesthetic induction chamber. Body length was determined with a scaled ruler in $\mathrm{mm}$ from the nose tip to the last hairs of the tail base.

\section{Diet Intake}

Food consumption was measured by using special feeders, which allowed the recovery of spilled food. Food intake was weighed daily with a Mettler scale (accuracy $\pm 1 \mathrm{mg}$ ).

\section{Evaluation of Femur Morphology}

Femur length was measured with a digital calliper from the tip of the greater trochanter to the distal surface of the lateral-medial condyle. A Mettler PE 600 scale (Zurich, Switzerland) was used to measure femur weight expressed in $\mathrm{g}$.

\section{Histomorphometry}

Tibiae were resected, fixed in buffered formalin, decalcified $10 \%$ EDTA, $\mathrm{pH}$ maintained at 7 during 25 days, and were embedded in paraffin. Longitudinally oriented sections of the tibiae were obtained and stained with hematoxylineosin. Histomorphometric evaluation of the decalcified bone section was performed on digitalized microphotographs by employing Image-Tool software (University of Texas Health Science Centre at San Antonio, UTHSCA). The following static parameters were evaluated in the middle area of the subchondral bone [26]: PZTh $(\mu \mathrm{m})$ : Thickness of proliferative zone of growth plate cartilage; HpZTh $(\mu \mathrm{m})$ : Thickness of hypertrophic zone. The thickness of the proliferative and hypertrophic zone of growth plate was calculated as the mean of thirteen different measurements performed at thirteen locations randomly chosen on each section; BV/TV (\%): Bone volume: fraction of bone tissue in total volume. Total volume was taken as bone tissue plus bone marrow.

\section{Biomechanical Tests on Femur}

The whole bones were submitted to a three-point bending test in a computerized Instron Universal Testing Machine (model 4442; Canton, MA, USA). In this procedure of bone breaking, the force was delivered to the midshaft by a crosshead at a constant speed. The breaking force was applied perpendicularly to the long axis of the bone at midshaft. Bones were placed lying horizontally with the anterior aspect facing down on two supports equidistant from their ends, separated by a constant distance, and loaded $(50 \mathrm{~N})$ centrally at a speed of $5 \mathrm{~mm} / \mathrm{min}$. Femurs were broken from the anterior to posterior plane. The plots of load v. deformation (Wd) obtained were analyzed to determine bone mechanical properties. These plots recorded both the elastic (linear) and plastic components, separated by the yield point. The following structural (whole-bone) mechanical properties were de- 
termined: Load-bearing capacity or fracture load (Wf, N), Yielding load (Wy, N), Yielding deformation (dy), Bone stiffness in elastic conditions or load-to-deformation ratio (Wy/dy, N/mm). Because bone segments between the supports were closely comparable to hollow, elliptically shaped cylinders, the micromorphometry of the horizontal and vertical external ( $\mathrm{H}$ and $\mathrm{V}$ ) and internal ( $\mathrm{h}$ and $\mathrm{v}$ ) diameters of the fracture sections enabled us to calculate the following geometric properties: Cross-sectional cortical bone area (A, $\mathrm{mm}^{2}$ ) and second moment of inertia of the cross-section in relation to the horizontal axis (Ix, $\mathrm{mm}^{4}$ ).

A stress-strain curve was determined from the previous force-deformation curve using engineering formulae [27]. Material properties calculated were: Yield stress ( $\delta \mathrm{y}$, $\mathrm{N} / \mathrm{mm}^{2}$ ) and Young's modulus of elasticity $\left(\mathrm{E}, \mathrm{N} / \mathrm{mm}^{2}\right)$.

\section{Hypothalamic Noradrenergic Neurotransmission Studies}

\section{Neuronal NE Uptake}

After animals were decapitated, between 9:00 and 12:00 am, hypothalami were removed and $\mathrm{AH}, \mathrm{MH}$ and $\mathrm{PH}$ were dissected according to Palkovits and Brownstein [28]. Tissues were pre-incubated in a Dubnoff incubator for $30 \mathrm{~min}$ at $37^{\circ} \mathrm{C}$ in Krebs bicarbonate solution supplemented with minimum essential media amino acid solution and basal medium Eagle vitamin solution (KSS; MBP Biomedicals, USA), $\mathrm{pH} 7.4$, and bubbled with a gas mixture $\left(95 \% \mathrm{O}_{2}\right.$ and $5 \% \mathrm{CO}_{2}$ ) under continuous shaking. $\left[{ }^{3} \mathrm{H}\right] \mathrm{NE}$ uptake was measured according to the method described by Vatta et al. [29] with minor modifications. Briefly, tissues were incubated with $100 \mathrm{nM}$ dl- $\left[7,8-{ }^{3} \mathrm{H}\right] \mathrm{NE}(9.75 \mathrm{Ci} / \mathrm{mmol}$ of specific activity; Perkin Elmer Life Sciences Inc., MA, USA). During preincubation and incubation periods, monoamine-oxidase (MAO) activity and extraneuronal NE uptake were inhibited by the addition of $50 \mu \mathrm{M}$ pargyline and $100 \mu \mathrm{M}$ hydrocortisone, respectively. Then, tissues were washed for $5 \mathrm{~min}$. (6 times) with cold KSS, followed by homogenization with $0.4 \mathrm{~N} \mathrm{HClO}_{4}$ and centrifugation. The activity of ${ }^{3} \mathrm{H}$ corresponding to NE was determined in the supernatant by usual scintillation counting methods. Results were expressed as percentage of $\mathrm{C}$ group $\pm \mathrm{SEM}$.

\section{Neuronal NE Release}

The experimental procedure was similar as described above except that in the last wash, $10 \mu \mathrm{M}$ desipramine was added in order to inhibit neuronal NE uptake. Then, 2 consecutive samples of incubation medium were collected every 5 min., and $\left[{ }^{3} \mathrm{H}\right]$ NE release was measured in each period as the amount of radioactivity present in the incubation medium. Results were expressed as percentage of the ratio between the second and first incubation period \pm SEM.

\section{TH Activity}

TH activity was determined by the methods of Reinhard [30]. Briefly, hypothalamic regions were obtained as described above, and homogenized in $500 \mu$ l buffer $(5 \mathrm{mM}$ $\mathrm{KH}_{2} \mathrm{PO}_{4}$ and $0.2 \%$ Triton $\mathrm{X}-100, \mathrm{pH} 7.0$ ). The samples were divided in two aliquots, one for protein determination and the other centrifuged for $10 \mathrm{~min}$. at $10.000 \mathrm{~g}$ at $4^{\circ} \mathrm{C}$ and an aliquot of supernatant incubated for $20 \mathrm{~min}$. at $37^{\circ} \mathrm{C}$ with $50 \mathrm{mM}$ HEPES ( $\mathrm{pH} 7.0)$ in the presence of $15 \mathrm{nmol} \mathrm{L-}$ tyrosine containing $0.5 \mu \mathrm{Ci} \quad \mathrm{L}-\left[3,5-{ }^{3} \mathrm{H}\right]$ tyrosine $(54.0$
$\mathrm{Ci} / \mathrm{mmol}$ of specific activity, GE Healthcare, UK), $420 \mathrm{mM}$ $\beta$-mercaptoethanol, $1000 \mathrm{U}$ catalase, and $0.75 \mathrm{mM}$ 6-methyltetrahydrobiopterin. The reaction was stopped by the addition of $1 \mathrm{ml}$ of $7.5 \%$ activated charcoal suspension in $1 \mathrm{~N}$ $\mathrm{HCl}$, and the final mixture was vortexed and centrifuged at $500 \mathrm{~g}$ for $10 \mathrm{~min}$. The supernatant was separated to determine the radioactivity of ${ }^{3} \mathrm{H}_{2} \mathrm{O}$ by conventional scintillation methods. Results are expressed as the percentage of $\mathrm{C}$ group \pm SEM.

\section{Statistical Analysis}

Results are expressed as mean \pm standard error of the mean (SEM). Data were analysed by one-way ANOVA followed by the Student-Neuman-Keuls test. Differences were considered significant if $P<0.05$ [31]. Statistical analysis was performed with the Graphpad Prism (version 3.0) statistical package (Graphpad Software, San Diego, CA, USA).

\section{RESULTS}

As shown in Table 1a food restriction induced a highly significant $(P<0.001)$ negative effect in body growth in ND rats. After 4 weeks of food restriction, ND rats' body weight and length were $40.6 \%$ and $76.0 \%$, respectively, as compared to $\mathrm{C}$ rats. There was a catch-up growth during refeeding that reached the same values as $\mathrm{C}$ animals at week 12 (Table 1b). Femoral growth was negatively affected $(P<0.001)$ in ND rats at the end of the restrictive period (4 weeks) (Table 1a). During refeeding, no significant differences between ND and C rats were observed at week 12 of the experimental period (Table $\mathbf{1 b}$ ).

Table 2a and $\mathbf{2 b}$ show the histomorphometric evaluation on longitudinally oriented sections of the tibiae in ND and C groups at W4 and W12, respectively. At W4, thickness of proliferative and hypertrophic zones of growth plate cartilage and bone volume was $19.5,29.3,34.2 \%$ less $(P<0.05)$, respectively, as compared to C group (Table 2a). All parameters normalized with refeeding (Table $\mathbf{2 b}$ ).

As previously reported [16], structural properties, i.e. load-bearing capacity (Wf), yielding load (Wy) and diaphyseal stiffness $(\mathrm{Wy} / \mathrm{dy})$ were negatively affected in ND rats after food restriction $(P<0.001)$. However, after refeeding, ND structural parameters achieved $\mathrm{C}$ values. Nevertheless, significant differences in material bone quality, assessed by yield stress $(\delta y)$ and modulus of elasticity (E), were not evident between groups, at any time. Cross-sectional cortical area (A) and moment of inertia of the fracture section (Ix) were significantly reduced $(P<0.001)$ after 4 weeks of food restriction in the post-weaning period as compared to $\mathrm{C}$ rats. The correlation between Ix and A v. body weight was $\mathrm{r}^{2} 0.78$ and 0.67 , respectively. A high correlation was also found between Wf and Wy v. Ix ( $\mathrm{r}^{2} 0.72$ and 0.64 , respectively). In agreement with the effects on whole bone, no significant differences were observed in geometrical properties between groups at week 12 (Table 3a and 3b).

Hypothalamic noradrenergic neurotransmission studies are shown in Figs. (1-3). After the first 4 weeks of the experimental period, both neuronal uptake and release of NE showed a significant reduction in anterior hypothalamus of ND rats as compared with $\mathrm{C}$ groups (20.95 and $33.00 \%$, respectively), while TH activity was increased (64.26\%). At the same time, neuronal NE uptake was reduced (19.35\%) 
Table 1a. Body and femoral weight and length at week 4 after weaning for control (C) and experimental (ND) rats. Values are means with standard errors of the mean (SEM) for ten animals per group. Mean value is significantly different from that of the control at the same time point: $* * * P<0.001 ; * * P<0.01 ; * P<0.05$.

\begin{tabular}{|c|c|c|c|c|}
\hline & \multicolumn{3}{|c|}{ Week 4 } & \multicolumn{2}{c|}{ ND } & \multicolumn{2}{|c|}{ Mean } \\
\hline & & C & SEM & 73.80 \\
\hline \hline Mean & 182.00 & 8.36 & 15.02 & $7.07^{* * *}$ \\
\hline Body length (cm) & 19.80 & 0.35 & 0.30 & $0.58^{* * *}$ \\
\hline Femur weight (g) & 0.50 & 0.01 & 23.09 & $0.01^{* *}$ \\
\hline Femur length (mm) & 27.00 & 0.26 & $0.29 *$ \\
\hline
\end{tabular}

Table 1b. Body and femoral weight and length at week 12 after weaning for control (C) and experimental (ND) rats. Values are means with standard errors of the mean (SEM) for ten animals per group

\begin{tabular}{|c|c|c|c|c|}
\hline & \multicolumn{2}{|c|}{ Week 12 } \\
\hline & \multicolumn{2}{|c|}{ C } & SEM & \multicolumn{2}{c|}{ Mean } & SEM \\
\hline & Mean & 7.85 & 342.98 & 23.54 \\
\hline \hline Body weight (g) & 360.77 & 0.34 & 0.80 & 0.45 \\
\hline Body length (cm) & 24.55 & 0.04 & 34.00 & 0.03 \\
\hline Femur weight (g) & 0.90 & 0.38 & 0.45 \\
\hline Femur length (mm) & 34.50 & & 3 \\
\hline
\end{tabular}

Table 2a. Thickness of the proliferative and hypertrophic zone of growth plate and bone volume at week 4 after weaning for control (C) and experimental (ND) rats. Values are means with standard errors of the mean (SEM) for ten animals per group. Mean value is significantly different from that of the control at the same time point: $* P<0.01 ; * * P<0.05$

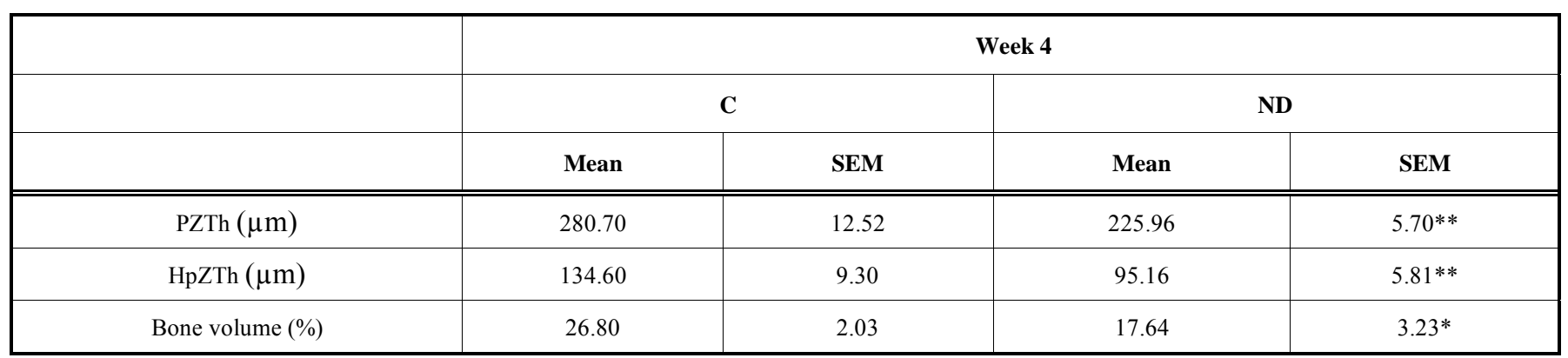

Table 2b. Thickness of the proliferative and hypertrophic zone of growth plate and bone volume at week 12 after weaning for control (C) and experimental (ND) rats. Values are means with standard errors of the mean (SEM) for ten animals per group

\begin{tabular}{|c|c|c|c|c|}
\hline & \multicolumn{4}{|c|}{ Week 12} \\
\hline & Mean & SEM & Mean & SEM \\
\hline $\operatorname{PZTh}(\mu \mathrm{m})$ & 173.57 & 5.71 & 180.00 & 4.28 \\
\hline $\operatorname{HpZTh}(\mu \mathrm{m})$ & 106.43 & 4.28 & 112.14 & 5.00 \\
\hline
\end{tabular}


Table 3a. Structural, material and geometrical properties of the femoral diaphysis at week 4 after weaning for control (C) and experimental (ND) rats. Values are means with standard errors of the mean (SEM) for ten animals per group. Mean value is significantly different from that of the control at the same time point: ***P<0.001

\begin{tabular}{|c|c|c|c|c|}
\hline & \multicolumn{2}{|c|}{ Week 4 } & \multicolumn{2}{c|}{ ND } \\
\hline & Mean & SEM & Mean \\
\hline & 48.03 & 2.67 & 27.61 & $1.02^{* * *}$ \\
\hline \hline $\mathrm{Wf}(\mathrm{N})$ & 42.89 & 1.07 & 22.07 & $0.98^{* * *}$ \\
\hline $\mathrm{Wy}(\mathrm{N})$ & 110.27 & 2.69 & 62.99 & $3.67 * * *$ \\
\hline $\mathrm{Wy} / \mathrm{dy}(\mathrm{N} / \mathrm{mm})$ & 897.64 & 91.30 & 979.70 & 70.50 \\
\hline $\mathrm{E}\left(\mathrm{N} / \mathrm{mm}^{2}\right)$ & 50.75 & 3.26 & 53.60 & 3.18 \\
\hline$\delta \mathrm{y}\left(\mathrm{N} / \mathrm{mm}^{2}\right)$ & 4.34 & 0.19 & 2.63 & $0.20^{* * *}$ \\
\hline $\mathrm{A}\left(\mathrm{mm}^{2}\right)$ & 2.87 & 0.13 & 1.24 & $0.08^{* * *}$ \\
\hline $\mathrm{Ix}\left(\mathrm{mm}^{4}\right)$ & & & \\
\hline
\end{tabular}

Table 3b. Structural, material and geometrical properties of the femoral diaphysis at week 12 after weaning for control (C) and experimental (ND) rats. Values are means with standard errors of the mean (SEM) for ten animals per group

\begin{tabular}{|c|c|c|c|c|}
\hline & \multicolumn{4}{|c|}{ Week 12} \\
\hline & \multicolumn{2}{|c|}{ C } & \multicolumn{2}{|c|}{ ND } \\
\hline $\mathrm{Wf}(\mathrm{N})$ & 129.82 & 4.83 & 122.07 & 28.60 \\
\hline Wy $(\mathrm{N})$ & 109.98 & 13.52 & 103.48 & 5.23 \\
\hline $\mathrm{E}\left(\mathrm{N} / \mathrm{mm}^{2}\right)$ & 1027.60 & 83.04 & 1071.62 & 129.75 \\
\hline$\delta y\left(N / m^{2}\right)$ & 93.58 & 6.32 & 89.52 & 5.23 \\
\hline $\mathrm{A}\left(\mathrm{mm}^{2}\right)$ & 5.23 & 0.29 & 5.52 & 0.28 \\
\hline
\end{tabular}

whereas neuronal NE release and TH activity were increased (47.00 and $52.42 \%$, respectively) in the posterior hypothalamus of experimental group as compared to $\mathrm{C}$ rats. No differences were observed in medial hypothalamus between groups at W4 and in all hypothalamic regions (anterior, medial and posterior) at week 12 .

\section{DISCUSSION}

The nutritional stress model used herein was developed in weanling male rats in our laboratory [8] and resembled the sub-optimal nutrition observed in children who consume inappropriate diets with insufficient total energy to sustain normal growth and weight gain $[9,10]$.

As it was previously demonstrated [16], the food restriction imposed was severe enough to decrease normal growth rate in ND animals. This negative effect was more evident in body weight than in body length. These observations may result from the combined effect of mild sub-optimal intake in addition to the length of the experimental period. A similar behavior was observed in the femur for the experimental group after 4 weeks of food restriction. However, when restriction ceased, complete catch-up [32] of the mentioned parameters was observed in ND rats.

Many factors are determinants of bone quality in both the axial and appendicular skeleton, but nutritional status is the most important. Protein-energy malnutrition during development, mostly during critical periods of body growth, contributes to longitudinal growth failure with subsequent risk of osteoporosis and bone fragility later in life [16, 33]. It is well known that restriction of energy intake reduces bone formation in both malnourished children [34] and undernourished rats [35, 36].

Although there is evidence of compensatory mechanisms in energy metabolism and body composition in response to mild chronic food restriction [8], both animal and human studies indicate that protein-energy malnutrition can be detrimental for both acquisition of bone mass during growth and its conservation during adulthood. Therefore, malnutri- 
tion during development can lead to impairment of biomechanical competence $[16,33,37]$. In fact, in the present study, detrimental effects of mild chronic food restriction on bone were evident in histomorphometric parameters in ND rats. In tibiae, histomorphometric studies showed that thickness of proliferative and hypertrophic zones of growth plate cartilage and bone volume were negatively affected in ND rats after food restriction as compared to $\mathrm{C}$ rats.

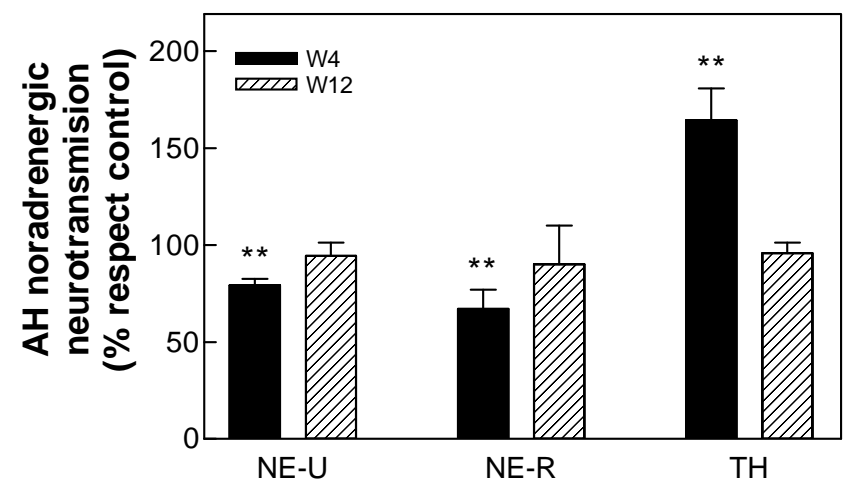

Fig. (1). Anterior hypothalamic noradrenergic neurotransmision (\% respect control) at different times (W4 and W12) after weaning for ND rats. Values are means with standard errors of the mean (SEM) for ten animals per group. Mean value is significantly different from that of the control at the same time point: $* * P<0.01$; * $P<0.05$. NE-U: neuronal norepinephrine uptake; NE-R: neuronal norepinephrine release; TH: tyrosine hydroxylase activity; W4: week 4; W12: week 12.

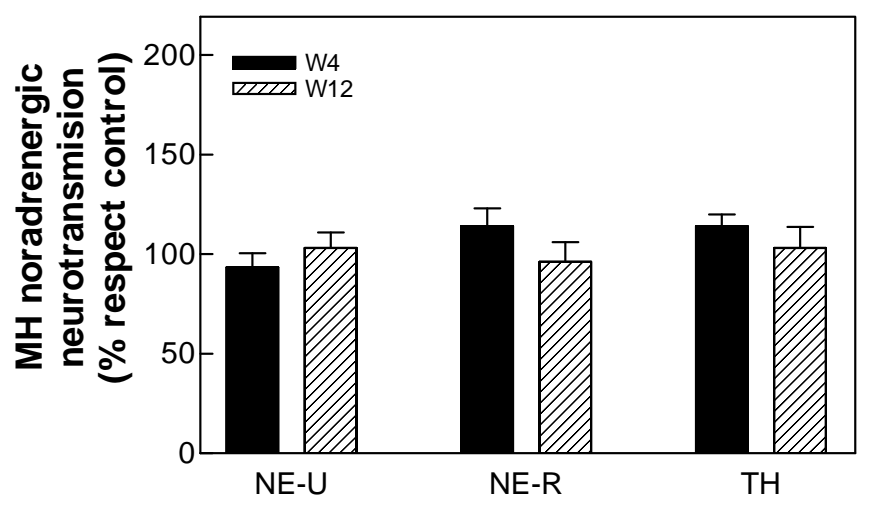

Fig. (2). Medial hypothalamic noradrenergic neurotransmission (\% respect control) at different times (W4 and W12) after weaning for ND rats. Values are means with standard errors of the mean (SEM) for ten animals per group. Mean value is not significantly different from that of the control at the same time point. NE-U: neuronal norepinephrine uptake; NE-R: neuronal norepinephrine release; TH: tyrosine hydroxylase activity; W4: week 4; W12: week: 12.

It is well known that the mechanical properties of bone as a whole are determined by both geometry and material properties [27]. Changes in the structural properties of a solid body of bone could be due to changes in the mass and its spatial distribution and/or intrinsic mechanical quality of its constitutive substance [38]. As previously seen in our laboratory [16] when load-deformation curves obtained were analysed, a negative effect in mechanical strength of diaphyseal femurs (Wf, Wy) was evident in response to external loading after 4 weeks of food restriction in the post-weaning period. In congruence with mechanical properties, the crosssectional parameters, A and Ix, were significantly reduced in ND rats when compared with control animals of the same age. On the other hand, the material quality of bone $(\delta y, E)$ was not affected by food restriction, in agreement with other studies [39]. The lack of difference in material properties between ND and $\mathrm{C}$ animals after food restriction suggests that the impaired performance of diaphyseal shafts of ND animals should be regarded as resulting predominantly from changes in the spatial distribution of bone material, rather than its intrinsic quality. All parameters assessed normalized according to catch-up growth, allowing appropriate bone mechanical competence of the appendicular skeleton to be attained during refeeding.

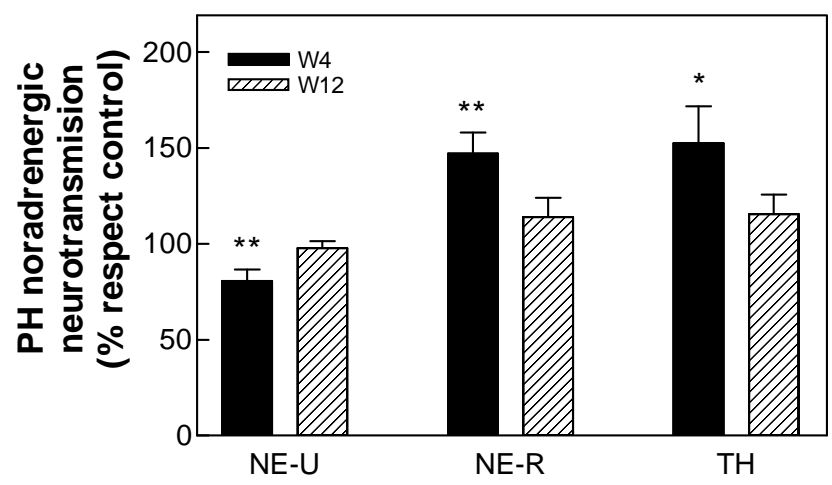

Fig. (3). Posterior hypothalamic noradrenergic neurotransmission (\% respect control) at different times (W4 and W12) after weaning for ND rats. Values are means with standard errors of the mean (SEM) for ten animals per group. Mean value is significantly different from that of the control at the same time point: ** $P<0.01$; * $P<0.05$. NE-U: neuronal norepinephrine uptake; NE-R: neuronal norepinephrine release; TH: tyrosine hydroxylase activity; W4: week 4; W12: week 12.

Considering hypothalamus as a centre involved in physiological, metabolic, autonomic, neuroendocrine and behavior functions, many studies have demonstrated that malnutrition during childhood and youth could result in different morpho-functional brain dysfunctions whose severity depends on the time of onset, duration and intensity of suboptimal and/or unbalanced feeding [40].

Results obtained on neuronal NE uptake and release as well as TH activity in the anterior hypothalamus, suggest a reduced noradrenergic activity in this sympathoinhibitory brain region in ND rats after food restriction. These findings may result from diminished leptin serum levels in the present nutritional stress model as it was previously demonstrated [11]. There is considerable evidence that indicates that leptin may increase sympathetic nerve signals to brown adipose tissue, kidneys and adrenal glands, with unequivocal sympathoexcitatory actions in rodents $[41,42]$.

In the anterior hypothalamus, the minor NE release observed in ND rats results in a less quantity of catecholamine in the synaptic cleft, and therefore, less postsynapsis stimulation. The reduced NE uptake in this area may arise from de- 
creased NE release and/or a less affinity in the carriermediated process, which is the most important mechanism through which NE ends its effects in the synaptic gap [43].

After food restriction, TH activity in ND rats seems to be controversial as compared to the other catecholaminergic parameters analyzed. The regulation of TH is very complex and involves diverse factors that affect catecholamine release. TH is a specific marker for catecholaminergic neurons, catalyzes the hydroxylation of L-tyrosine to L-DOPA, being the rate-limiting step in the production of catecholamines $[44,45]$. The increment in TH activity in the ND group may result from the activity of the enzyme in other catecholaminergic neurons such as the dopaminergic [46]. It is important to point out that we assessed total TH activity and not only the activity of the enzyme in norepinephrine containing neurons.

In medial hypothalamus, no significant differences were observed in ND rats after food restriction as compared to $\mathrm{C}$ group. These findings could be the result of a great variety of neuronal input and/or humoral influences on noradrenergic neurons without evident sympathetic changes.

Regarding NE activity in the posterior hypothalamic region (sympathoexitatory area), once sub-optimal nutrition ended, an increase in catecholaminergic activity (augmented NE release and TH activity, and reduced NE uptake) was observed in ND rats as compared with $\mathrm{C}$ animals. Present findings are in accordance with an increase in NE biosynthesis and a higher postsynaptic stimulation in the present nutritional stress model.

\section{CONCLUSION}

In conclusion, global sympathetic hypothalamic activity is enhanced, supported by diminished catecholaminergic activity in the anterior hypothalamus, is considered a sympathoinhibitory centre, and increased catecholaminergic activity in the posterior hypothalamus, considered a sympathoexcitatory centre [47, 48]. Present findings suggest that in our nutritional stress model, impaired biomechanical bone performance could be due, at least in part, to the overall increase of hypothalamic noradrenergic activity in response to mild chronic food restriction. Refeeding caused a reversal of the deleterious effects of food restriction on growth, bone parameters and global sympathetic hypothalamic activity in ND rats, suggesting that mild chronic food restriction does not produce long-term side-effects on bone appendicular skeleton in response to adaptive mechanisms developed by undernourished rats in order to face nutritional stress.

\section{ACKNOWLEDGEMENTS}

The Authors thank Ricardo H Orzuza for his assistance in animal care.

This work was supported by research grants from the University of Buenos Aires (UBACyT O010 and O011).

\section{REFERENCES}

[1] Friedman SM, Rodriguez PN, Boyer PM, Lifshitz F. Decreased energy expenditure- an adaptive mechanism of nutritional growth retardation. Nutr Res 2006; 26: 345-9.

[2] Lifshitz F, Moses N. Growth failure: a complication of dietary treatment of hypercholesterolemia. Am J Dis Child 1989; 143: 53742.
[3] Pugliese MT, Weyman-Daum M, Moses N, Lifshitz F. Parental health beliefs as a cause of nonorganic failure to thrive. Pediatrics 1987; 80: 175-82.

[4] Lifshitz F, Moses N. Nutritional dwarfing: growth, dieting and fear of obesity. J Am Coll Nutr 1988; 7: 367-76.

[5] Keller W, Fillmore CM. Prevalence of protein-energy malnutrition. World Health Stat Q 1983; 36: 129-67.

[6] Lifshitz F, Moses N, Cervantes C, Ginsberg L. Nutritional dwarfing in adolescents. Semin Adolesc Med 1987; 3: 255-66.

[7] Sandberg DE, Smith MM, Fornari V, Goldstein M, Lifshitz F. Nutritional dwarfing: is it a consequence of disturbed psychosocial functioning? Pediatrics 1991; 88: 926-33.

[8] Friedman SM, Rodriguez PN, Olivera MI, et al. Enanismo por desnutrición: cronodinamia de los procesos metabólicos en ratas. Medicina (B Aires) 1998; 58: 282-86.

[9] Kaplan RM, Toshima MT. Does a reduced fat diet cause retardation in child growth? Prev Med 1992; 21: 33-52.

[10] Akeson PK, Axelsson IEM, Raiha NCR, Warm A, Minoli I, Moro G. Fat intake and metabolism in Swedish and Italian infants. Acta Paediatr 2000; 89: 28-33.

[11] Compagnucci CV, Compagnucci GE, Lomniczi A, et al. Effect of nutritional stress on the hypothalamo-pituitary-gonadal axis in the growing male rats. Neuroimmunomodulation 2002-03; 10: 153-62.

[12] Cheung CC, Thornton JE, Nurani SD, Clifton DK, Steiner RA. A reassessment of leptin's role in triggering the onset of puberty in the rat and mouse. Neuroendocrinology 2001; 74: 12-21.

[13] Urbanski HF. Leptin and puberty. Trends Endocrinol Metab 2001; 12: 428-29.

[14] Kennedy GC, Mitra J. Body weight and food intake as initiating factors for puberty in the rat. J Physiol 1963; 166: 408-18.

[15] Flier JS. Clinical review 94: What's in a name? In search of leptin's physiologic role. J Clin Endocrinol Metab 1998; 83: 140713.

[16] Boyer PM, Compagnucci GE, Olivera MI, et al. Bone status in an animal model of chronic sub-optimal nutrition: a morphometric, densitometric and mechanical study. Br J Nutr 2005; 93: 663-9.

[17] Compagnucci GE, Compagnucci CV, Olivera MI, et al. Estudio comparativo morfométrico, densitométrico y biomecánico de fémur y mandíbula en un modelo animal de enanismo por desnutrición. Rev Argent Osteol 2005; 4: 10-22.

[18] Chrousos GP. Stress, chronic inflammation, and emotional and physical well-being: concurrent effects and chronic sequelae. J Allergy Clin Immunol 2000; 106: S275-91.

[19] Bernardis LL, Bellinger LL. The lateral hypothalamic area revisted: Neuroanatomy, body weight regulation, neuroendocrinology and metabolism. Neurosci Biobehav Rev 1993; 17: 141-93.

[20] Rondeel JMM, Jackson IMD. Molecular biology of the regulation of hypothalamic hormones. J Endocrinol Invest 1993; 16: 219-46.

[21] Wardener HE. The hypothalamus and hypertension. Physiol Rev 2001; 81: 1599-658

[22] Landsberg L. Feast or famine: the sympathetic nervous system response to nutrient intake. Cell Mol Neurobiol 2006; 26: 497-508.

[23] Tannenbaum GS, Gurd W, Lapointe M. Leptin is a potent stimulator of spontaneous pulsatile growth hormone $(\mathrm{GH})$ secretion and the GH response to GH-releasing hormone. Endocrinology 1998; 139: 3871-5.

[24] Pombo M, Pombo CM, Astorga R, et al. Regulation of growth hormone secretion by signals produced by the adipose tissue. J Endocrinol Invest 1999; 22: 22-6.

[25] National Institutes of Health. Laboratory animal welfare: Public Health Service policy on humane care and use of laboratory animals by awardee institutions; 1985, revised 1990 .

[26] Parfitt AM, Drezner MK, Glorieux FH, et al. Bone histomorphometry: standardization of nomenclature, symbols, and units. Report of the ASBMR Histomorphometry Nomenclature Committee. J Bone Miner Res 1987; 2: 595-610.

[27] Turner CH, Burr DB. Basic biomechanical measurements of bone: a tutorial. Bone 1993; 14: 595-608.

[28] Palkovits M, Brownstein MJ. Maps and Guide to Microdissection of the Rat Brain, Elsevier, New York, Amsterdam, London; 1988.

[29] Vatta MS, Presas M, Bianciotti LG, Zarrabeita V, Fernández BE. B and $\mathrm{C}$ types natriuretic peptides modulate norepinephrine uptake and release in the rat hypothalamus. Regul Pept 1996; 65: 175-84.

[30] Reinhard JF, Smith GK, Nichol CA. A rapid and sensitive assay for tyrosine-3 monooxigenase based upon the release of ${ }^{3} \mathrm{H}_{2} \mathrm{O}$ and absorption of $\left[{ }^{3} \mathrm{H}\right]$-tyrosine by charcoal. Life Sci 1986; 118: 2185-9. 
[31] Sokal R, Rohlf J. Biometry: The Principles and Practice of Statistics in Biological Research. San Francisco, CA: WHFreeman\&Co; 1994.

[32] Boersma B, Wit JM. Catch-up growth. Endocr Rev 1997; 18: 64661.

[33] Bonjour JP, Ammann P, Chevalley T, Rizzoli R. Protein intake and bone growth. Can J Appl Physiol 2001; 26: 153-66.

[34] Ndiaye B, Lemonnier D, Sall MG, et al. Serum osteocalcin regulation in protein-energy malnourished children. Pediatr Res 1995; 37: 606-10.

[35] Ndiaye B, Cournot G, Pelissier M, Debray OW, Lemonnier D. Rat serum osteocalcin is decreased by restriction of energy intake. $\mathrm{J}$ Nutr 1995; 125: 1283-90.

[36] Rhee Y, Namgung R, Park DH, Lee HC, Huh GB, Lim SK. The effects of recombinant human parathyroid hormone, rhPTH(1-84), on bone mass in undernourished rats. J Endocrinol 2002; 174: 41925.

[37] Alippi RM, Meta MD, Bozzini C, Olivera MI, Ferretti JL, Bozzini CE. Dynamics of recovery of morphometrical variables and $\mathrm{pQCT}$ derived cortical bone properties after a short-term protein restriction in maturing rats. Growth Dev Aging 2001; 65: 67-72.

[38] Ferretti JL. Biomechanical properties of bone. In Osteoporosis and Bone Densitometry, [HK Gennant, G Guglielmi and M Jergas, editors]. Berlin: Springer Verlag; 1997; pp. 143-61.

[39] Ferretti JL, Capozza R, Cointry G, Bozzini C, Alippi RM, Bozzini $\mathrm{CE}$. Additive effects of dietary protein and energy deficiencies on diaphysis and bone tissue of rat femurs as determined by bending tests. Acta Physiol Pharmacol Ther Latinoam 1991; 41: 253-62.
[40] Soto-Moyano R, Belmar J, Perez H, Ruiz S, Hernandez A. Central noradrenergic hyperactivity early in life: a hypothesis on the origin of morpho-functional brain disorders induced by malnutrition. Biol Res 1995; 28: 105-11.

[41] Dunbar JC, Hu Y, Lu H. Intracerebroventricular leptin increases lumbar and renal sympathetic nerve activity and blood pressure in normal rats. Diabetes 1997; 46: 2040-3.

[42] Aizawa-Abe M, Ogawa Y, Masuzaki H, et al. Pathophysiological role of leptin in obesity-related hypertension. J Clin Invest 2000; 105: $1243-52$.

[43] Mandela P, Ordway GA. The norepinephrine transporter and its regulation. J Neurochem 2006; 97: 310-33.

[44] Kaufman S. Tyrosine hydroxylase. Adv Enzymol Relat Areas Mol Biol 1995; 70: 103-220.

[45] Kumer SC, Vrana KE. Intrincate regulation of tyrosine hydroxylase activity and gene expression. J Neurochem 1996; 67: 443-62.

[46] Horvath TL, Naftolin F, Leranth C. Luteinizing hormone-releasing hormone and gamma-aminobutyric acid neurons in the medial preoptic area are synaptic targets of dopamine axons originating in anterior periventricular areas. J Neuroendocrinol 1993; 5: 71-9.

[47] Barman SM. Descending projections of hypothalamic neurons with sympathetic nerve-related activity. J Neurophysiol 1990; 64: 101932.

[48] Barron KW, Heesch CM. Cardiovascular effects of posterior hypothalamic stimulation in baroreflex-denervated rats. Am J Physiol 1990; 259: 720-7. 